\title{
INFLUENCE OF THICKNESSES OF DIFFERENT CERAMIC MATERIALS ON WATER SORPTION OF LIGHT CURED RESIN CEMENT (IN VITRO STUDY)
}

\author{
Tamer Affify ${ }^{1 *}$, Marwa El-Wahsh ${ }^{2}$, Maged Zohdy ${ }^{2}$
}

\begin{abstract}
Objectives: The aim of this research was to study the effect of different thicknesses of three ceramic materials on the water sorption of light cured resin cements. Materials and methods: A total of 60 ceramic disc specimens were divided into 3 groups $(n=20)$ according to the type of ceramic into group (E): E.max CAD, Group (V): Vita Suprinity and Group (C): Celtra Duo. Each group was subdivided into 2 subgroups of different thicknesses; $0.4 \mathrm{~mm}$ and $1 \mathrm{~mm}$ thickness. Opaque light cured Bisco resin cement specimen was made of thickness $0.1 \mathrm{~mm}$ controlled using a teflon mold and cured through each ceramic specimen then inserted into the dessicator inside an incubator. The specimens were repeatedly weighed after $24 \mathrm{~h}$ intervals using analytical balance of accuracy $0.001 \mathrm{~g}$ until a constant mass $(\mathrm{m} 1)$ was obtained. The water sorption for the seven days of storage in water was calculated and the data was collected, tabulated and statistically analyzed. Results: There was a significant difference between samples of different materials $(\mathrm{p}<0.001)$. The highest value was found with Emax samples $(32.52 \pm 3.05)$ followed by Celtra Duo $(24.88 \pm 2.35)$ while the lowest value was found with Vita Suprinity $(23.36 \pm 2.39)$ while There was no significant difference between samples made with different thicknesses $(\mathrm{p}=0.376)$. Conclusion: There is a relationship between ceramic type and water sorption between the zirconia reinforced lithium silicates and lithium disilicate ceramic materials. Also, different ceramic thicknesses up to $1 \mathrm{~mm}$ did not affect the water sorption of the light cured resin cement.
\end{abstract}

KEYWORDS: Water sorption, Resin cements, Ceramic thickness

\section{INTRODUCTION}

The use of ceramic materials has increased due to their natural appearance, fluorescence, biocompatibility, durability and many other unique characteristics. The success of a ceramic restoration is mainly based on a high bond strength on the adhesion complex formed between the ceramic, resin cement and dental hard tissues. Lithium disilicate (IPS e.max CAD, Ivoclar Vivadent) is nowadays very popular ceramic composed of approximately $70 \mathrm{Vol} \%$ of crystalline phase incorporated in glassy matrix. It is being widely used owing to combining outstanding aesthetic appearance with high strength in addition to biocompatibility, chemical stability, machinable and easy processing. In addition, lately, a zirconia reinforced lithium silicate glass ceramic has been introduced for dental CAD/CAM applications. It is used in similar indications for lithium disilicate ceramic including fabrication of inlays, onlays, partial crowns, veneers, anterior and posterior crowns and anterior and posterior single tooth restorations on implant abutments ${ }^{(1)}$.

1. Demenstrator, Fixed Prosthodontics Department, British University in Egypt, Cairo, Egypt

2. Associate Professor, Fixed Prosthodontics Department Faculty of Dentistry, Ain Shams University, Cairo, Egypt

-Corresponding author: tamer.aboelsoud@bue.edu.eg 
This new glass ceramic is enriched with zirconia ( $\approx 10 \%$ by weight). Manufacturers claimed that this newly developed generation of glass ceramic materials combines the positive material characteristics of zirconia ( $\mathrm{ZrO} 2)$ and glass ceramic $^{(2)}$. The zirconia particles are incorporated in order to reinforce the ceramic structure by crack interruption. It has been supposed that the structure which is obtained after crystallization, exhibits enhanced mechanical properties and fulfills the highest esthetic requirements. It is anatomically contoured as monolithic restoration due to enhanced translucency and different shades ${ }^{(3)}$. There is no doubt that the cementation process strongly impacts clinical success of ceramic restorations. Resin cements with their bonding ability to dental tissues and ceramics offer sufficient bond strength to get acceptable longevity of the restoration ${ }^{(4)}$.

In order to obtain high bond strength between the ceramic restoration and the resin cement with the tooth structure, an optimal curing of the luting materials is required. The degree of conversion of resin-based materials is based on the cross-linking of monomer units to form long chains (polymers). This mechanism ensures unique physical and mechanical properties for the composite resins and resin luting agents ${ }^{(4,5)}$. However, the polymerization reaction can be affected by extrinsic factors such as the shade and thickness of the indirect restoration, temperature, and polymerization lights, as well as by the amount of light energy received ${ }^{(6)}$. Light-cure (LC) cements are used under thin and translucent restorations where there is adequate light transmission. When the restoration thickness is above $1.5-2 \mathrm{~mm}$ or its opacity inhibits light transmission, the use of dualcure (DC) resin cements is advocated ${ }^{(7)}$.

Most dentists report that they routinely use LC cements for all anterior restorations and DC cements for all posterior restorations regardless of the properties of the ceramic restoration. Nevertheless, independent from the location of the restoration, the shade and thickness of the ceramic restoration may vary considerably. Incomplete polymerization of the cement may lead to color instability, toxicity from residual monomer, decreased bond strength, and post-operative sensitivity, leading to increased risk of microleakage, food impaction and caries ${ }^{(8)}$. Several techniques are currently used to assess the DC of resin based dental materials or at least to assure achieving and adequate level of cure. Many of these techniques provide real-time data allowing dynamic monitoring of the polymerization process. Other mechanisms are static tests and can be relatively simple in nature ${ }^{(9)}$. Properties such as water sorption and solubility of conventional or adhesive luting cements have been extensively studied where it was stated that resin cements exhibit less microleakage than the conventional ones, indicating increased long-term clinical durability ${ }^{(10)}$.

In oral environmental conditions, the luting cement around the margins of the restoration are constantly affected by fluids leading to dissolution of resin cement continuously. Few residual monomers elute into the oral cavity, with most of them remaining trapped in the hardened resin. This dissolution rate is initially rapid and decreases with time. Degradation of the filler-matrix interface may result from water absorption into the resin cement. It may also result in polymer swelling, plasticizing, glass transition temperature reduction, polymer network weakening, reduction of tensile strength, and wear resistance. Water sorption and solubility may result in stress-induced degradation of the luting cement causing debonding and/or fracture of the restoration, increased marginal leakage, and potentially recurrent caries ${ }^{(11)}$.

The released residual, unreacted monomers may lead to adverse biological reactions by entering the dentinal tubules into the pulp. Unreacted monomer is found trapped in the polymerized composite resin and may reduce the clinical viability of the material through oxidation and hydrolytic degradation, 
which may appear in the form of discoloration of the resin. Moreover, marginal defects may occur, which lead to microleakage, debonding, or recurrent caries. All these factors are crucial as they influence the long-term success of the restorations and their survival rate. Two main theories explain the way by which water diffuses in a resin-based material after its application into the oral cavity. The free volume theory stating that water diffuses through microvoids, resin-filler interfaces, and morphological defects with no reaction to polar group; and the second theory; interaction theory states that water molecules form hydrogen bonds with specific ionic groups of the polymer chain ${ }^{(12)}$.

In view of these considerations, there's a hypothesis that thickness of a ceramic will interfere with the polymerization of the cements to such a degree that it will change the water sorption and solubility of this material; similarly, the type of the cement might also influence the outcome. Thus, the objective of this study was to evaluate the water sorption of light-cured cements using ceramic slides of different thicknesses and different ceramic materials.

\section{SUBJECTS AND METHODS}

A total of 60 Specimens were divided into 3 groups (20 each) according to the type of ceramic into group (E): Lithium disilicate (IPS e-max) as a control, group (S): Zirconia-Reinforced Lithium Silicate [SUPRINITY® PC] and group (C): ZirconiaReinforced Lithium Silicate [Celtra ${ }^{\circledR}$ Duo] where in all groups $(n=20)$. Each group was subdivided into 2 subgroups according to thickness into subgroup (I): $0.4 \mathrm{~mm}$ sub group (II): $1 \mathrm{~mm}$. The sixty ceramic slices were machined from their respective blocks by using a low speed diamond saw (Buehler Isomet diamond saw 4000, Buehler, USA) to uniform standard thicknesses of $(0.4 \mathrm{~mm}$ and $1.0 \mathrm{~mm})$.
A digital caliper (Holex digital caliper, Hoffmann Group, Germany) was used to verify the thicknesses. Crystallization was done for both partially crystallized Lithium disilicate and partially crystallized zirconia reinforced Lithium silicate slices according to the manufacturer recommendations as to allow the immature crystals to reach the desired volume and dimension. As for the preparation of Resin Cement specimens, two teflon moulds were fabricated to ensure a standard thickness of resin cement samples with an external diameter of $20 \mathrm{~mm} \times 3 \mathrm{~mm}$ thickness. An inner dimension $14 \times 14$ square shape was cut with two different thicknesses $0.5 \mathrm{~mm}$ and $1.1 \mathrm{~mm}$ to accommodate the designated thicknesses of ceramic slices $0.4 \mathrm{~mm}$ and $1.0 \mathrm{~mm}$ respectively and to ensure a $0.1 \mathrm{~mm}$ uniform cement thickness. The ceramic slices were seated on the inner stopper of the mold.

The lower opening which is a square of $14 \mathrm{X} 14$ $\mathrm{mm}$ used for the placement of the ceramic disc and the resin cement (two thicknesses were fabricated for the different ceramic samples $0.5 \mathrm{~mm}$ and $1.1 \mathrm{~mm}$ to obtain a thickness of $0.1 \mathrm{~mm}$ for the resin cement) this distance is measured from the outer surface to the inner shoulder Figure (1\&2). Resin cement was dispensed from the syringe on the ceramic slices, a celluloid strip placed and then the glass slab was applied with pressure to ensure complete seating and uniform $0.1 \mathrm{~mm}$ thickness of cement created by the mold.

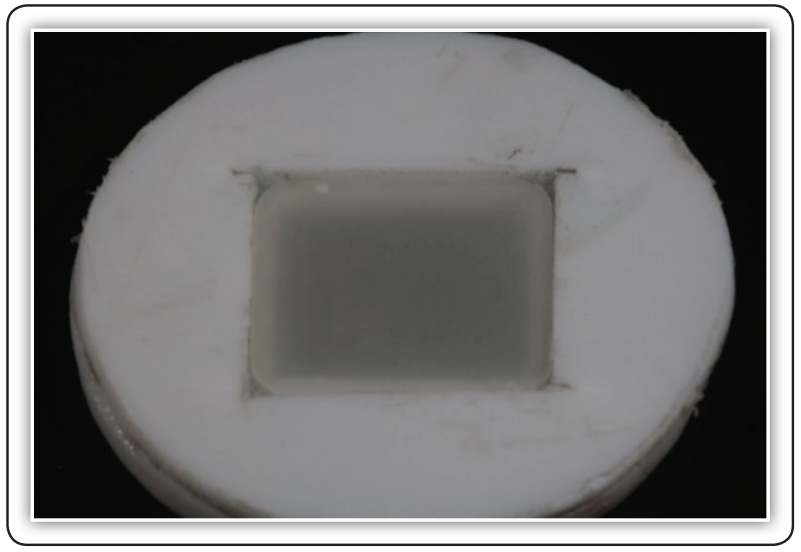

FIG (1) Teflon mould showing the internal stop 


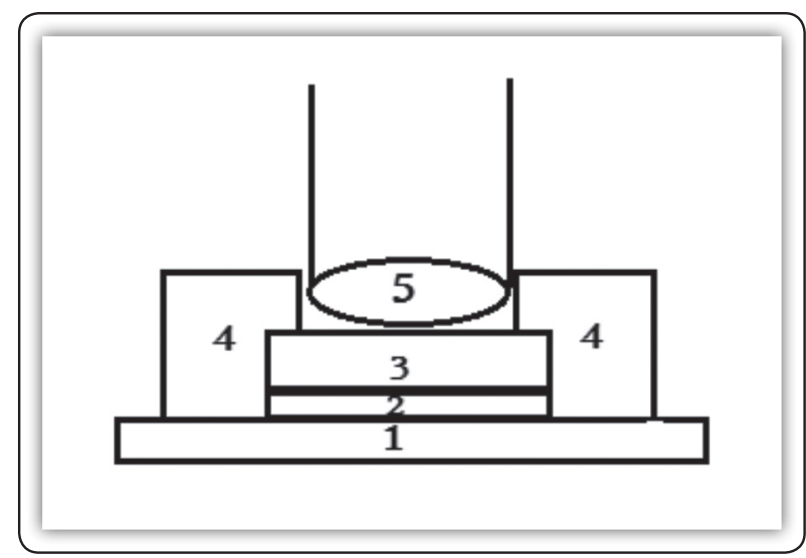

FIG (2) Diagrammatic representation of the resin/ceramic sample preparation:
1. Glass slab
2.Resin cement
3. Ceramic disk
4. Custom made Teflon mold
5. Tip of light curing unit

The glass slab was inverted and the upper opening was used for curing the resin cement through the ceramic slice, making the mold and ceramic slice underneath and LED curing light with intensity $1500 \mathrm{~mW} / \mathrm{cm}^{2}$ (Blue phase Light curing unit, Ascent PX, CAO group, USA) was used to cure the resin cement throughout all the samples for 20 seconds; while the tip of the curing unit was in direct contact with the ceramic slice. Water sorption was determined according to the ISO specification 4049. Immediately after polymerization, specimens were kept for twenty-four hours in a dark container to prevent further polymerization of the specimens and placed in a dessicator containing fresh blue silica gel (Shan dong shengpeng sodium silicate Co.,Ltd., China).The dessicator was then transferred to a preconditioning incubator at $37^{\circ} \mathrm{C}$.

The specimens were repeatedly weighed after $24 \mathrm{~h}$ intervals using analytical balance of accuracy $0.001 \mathrm{~g}$ until a constant mass (m1) was obtained (i.e., variation was less than $0.2 \mathrm{mg}$ in any $24 \mathrm{~h}$ period). Thickness, length and width of the specimens were measured using a digital caliber, rounded to the nearest $0.01 \mathrm{~mm}$, and these measurements were used to calculate the volume $(\mathrm{V})$ of each specimen (in $\mathrm{mm} 3$ ). Then, specimens were individually placed in sealed glass containers containing $10 \mathrm{ml}$ of distilled water $(\mathrm{pH} 7.2)$ at $37^{\circ} \mathrm{C}$ and placed in an incubator at $37^{\circ} \mathrm{C}$.

After 7 days of storage, the containers were removed from the incubator and left at room temperature for 30 minutes. The specimens were washed in running water, gently wiped with a soft absorbent paper, weighed in an analytical balance (m2). The specimens were dried inside a dessicator containing fresh silica gel and weighed daily until a constant mass (m3) was obtained The water sorption (WS) for the seven days of storage in water were calculated using the following formula:

$$
W S=\frac{m^{2}-m^{3}}{V}
$$

Where, $\mathrm{m} 1$ is the mass of the sample in micrograms before immersion in distilled water, $\mathrm{m} 2$ is the mass of the sample in micrograms after immersion in distilled water for seven days, $\mathrm{m} 3$ is the mass of the sample in micrograms after being conditioned in a dessicator with silica gel. Data were collected, tabulated and statistically analyzed.

\section{RESULTS}

As for the effect of Material on water sorption of the cement, there was a significant difference between samples of different materials $(p<0.001)$. The highest value was found with Emax samples (32.52 \pm 3.05$)$ followed by Celtra Duo $(24.88 \pm 2.35)$ while the lowest value was found with Vita Suprinity (23.36 \pm 2.39$)$. Pair wise comparisons showed Emax samples to have a significantly higher value than samples of other materials $(p<0.001)$. On the other hand, the effect of thickness showed no significant difference between samples made with different thicknesses $(p=0.376)$. The effect of thickness within each material reported that within all groups there was no significant difference between different thicknesses while for the effect of material within each thickness, for the $0.4 \mathrm{~mm}$ thickness, there was a significant difference between samples of different 
materials $(\mathrm{p}<0.001)$. The highest value was found with Emax samples (31.48 \pm 3.03$)$ followed by Celtra Duo $(25.78 \pm 2.13)$ while the lowest value was found with Vita Suprinity $(22.62 \pm 1.82)$. Pair wise comparisons showed samples of different materials to be significantly different from each other $(\mathrm{p}<0.001)$ and as for the $1 \mathrm{~mm}$ thickness, there was a significant difference between samples of different materials $(\mathrm{p}<0.001)$. The highest value was found with Emax samples (33.55 \pm 2.84 ) followed by Vita Suprinity $(24.10 \pm 2.75)$ while the lowest value was found with Celtra Duo (23.97 \pm 2.29$)$. Pair wise comparisons showed Emax samples to have a significantly higher value than samples of other materials $(\mathrm{p}<0.001)$.

TABLE (1) Mean \pm standard deviation (SD) of water sorption $(\mu \mathrm{g} / \mathrm{mm} 3)$ for different materials and thicknesses

\begin{tabular}{|c|c|c|c|l|}
\hline \multirow{2}{*}{ Thickness } & \multicolumn{3}{|c|}{ Material (mean \pm SD) } & \multirow{2}{*}{ p-value } \\
\cline { 2 - 5 } & Emax & $\begin{array}{c}\text { Vita } \\
\text { Suprinity }\end{array}$ & CeltraDuo & \\
\hline $0.4 \mathrm{~mm}$ & $31.48 \pm 3.03^{\mathrm{A}}$ & $22.62 \pm 1.82^{\mathrm{C}}$ & $25.78 \pm 2.13^{\mathrm{B}}$ & $<0.001^{*}$ \\
\hline $1 \mathrm{~mm}$ & $33.55 \pm 2.84^{\mathrm{A}}$ & $24.10 \pm 2.75^{\mathrm{B}}$ & $23.97 \pm 2.29^{\mathrm{B}}$ & $<0.001^{*}$ \\
\hline p-value & $0.071 \mathrm{~ns}$ & $0.194 \mathrm{~ns}$ & $0.113 \mathrm{~ns}$ & \\
\hline
\end{tabular}

Different superscript letters indicate a statistically significant difference within the same horizontal row*; significant $(\mathrm{p} \leq 0.05) \mathrm{ns}$; non-significant $(\mathrm{p}>0.05)$

\section{DISCUSSION}

In clinical practice, it is difficult to determine the thickness limit of a ceramic laminate veneer and/or translucency the material should possess to ensure reliable monomer conversion into polymers which make up the resin cement, especially when this conversion depends exclusively on the light energy deposited in the cement. The resin cements can be light-cured under lithium disilicate ceramic restorations of up to $2 \mathrm{~mm}$ thickness without impairing adhesion. However, studies show that increase in ceramic thickness results in lower transmittance of photo-curing light to the cementing agent and, consequently, the resin material presents decrease in hardness and monomer conversion. Moreover, the literature is not conclusive about which thickness variation limit and ceramic translucency would be optimal for proper transmittance of incident light. Therefore, thicknesses chosen in this study were $0.4 \mathrm{~mm}$ and $1 \mathrm{~mm}^{(13)}$.

Since ceramic thicknesses used in this study were 0.4 and $1 \mathrm{~mm}$, it was permitted to use light cured resin cements that allow sufficient light transmission to allow proper polymerization. In addition, light cured resin cement was preferred than dual cured owing to the latter's polymerization that involves free-radical initiators such as benzoyl peroxide that react with a tertiary amine. The amines form by-products during the polymerization reaction, which may cause a yellowish to brown discoloration, affecting the color stability of resin cements ${ }^{(14)}$.

In this study, cement thickness was adjusted to be $0.1 \mathrm{~mm}$ thickness which is considered as the maximum accepted thickness under ceramic laminate veneers. This cement thickness was controlled using a 2 custom made teflon molds adjusted to accommodate the 2 ceramic thicknesses included in this study. As stated by multiple authors, this determined cement thickness was mentioned to improve stress distribution under the veneers. A Teflon mold was fabricated to ensure standardization of the resin cement samples of even length, width and thickness and it was made-up according to the two thicknesses of ceramics used to regulate the thicknesses of cement used throughout the samples.

As proven by other studies, thickness of $0.1 \mathrm{~mm}$ for cement is suitable for fitting the veneer for better stress distribution between ceramic and cement interface. Also Zaghloul \& Mohsen and Runnacles et al used a cement thickness of $0.1 \mathrm{~mm}$ in their study to evaluate the degree of conversion of light cured cement through ceramic veneers ${ }^{(15,16)}$. 
The cured resin cement specimen were then placed in a dessicator containing fresh silica gel immediately after polymerization to measure water sorption. The dessicator is mandatory to ensure complete dehydration, removal of any remaining water in the specimens and prevent any water absorption from air humidity which was then transferred to a pre-conditioning incubator at $37^{\circ} \mathrm{C}$ to standardize the temperature and eliminate any variables. The containers were removed from the incubator after 7 days and left at room temperature for 30 minutes. Specimens were washed under running water, gently wiped with a soft absorbent paper and weighed with an analytical balance. The specimens were repeatedly weighed after $24 \mathrm{~h}$ intervals until a constant mass was obtained $(\mathrm{m} 2)$, followed by drying inside a dessicator containing fresh silica gel and weighed daily until a constant mass ( $m 3)$ was obtained ${ }^{(17)}$.

The water storage period of seven days, chosen for the present study, has been extensively studied. During seven days of storage in water the major components removed from the resinous materials are the residual monomers that have not reacted to form polymeric chains. The highest concentration of eluted monomers in 7 days was also observed by other authors ${ }^{(18,19)}$.

The null hypothesis of this study that stated that neither the type of material nor its thickness would affect the water sorption of the resin cement was rejected. The results of this study, concerning different material types, revealed that zirconia reinforced lithium silicate blocks caused significantly less water sorption of the underlying resin cement than that of lithium disilicate.

These results could be attributed to the microstructure of celtra duo where the inclusion of $10 \%$ zirconia dissolved into the lithium silicate glass matrix resulted in 4 times smaller silicate crystals, implying a high glass content and higher translucency than conventional lithium disilicate ceramics as stated by Awad et al ${ }^{(20)}$ and Maraghy et al. ${ }^{(21)}$ Increased translucency of celtra duo allowed increased passage of curing light improving the degree of conversion of the cement and consequently decreased its water sorption values. As for the Vita Suprinity zirconia reinforced glass ceramic, the current study showed that this material caused less water sorption for the underlying cement. This may be attributed to the higher degree of conversion of the cement under Vita Suprinity owing to it more translucent characteristic.

This conclusion came in agreement with Caprak et al (22) and Bahgat et al. (23) who reported vita Suprinity showed statistically significant higher mean translucency $(22.43 \pm 0.69)$ when compared to IPS emax CAD $(20.41 \pm 0.41)$ similar to the previous studies, he owed the results to the addition of zirconia and the ensuing nucleation process, resulting in more homogenous crystalline structure and finer crystal size $(0.5 \mu \mathrm{m})$ compared to the needle-shaped coarser crystalline structure $(1.5 \mu \mathrm{m})$ of lithium disilicate glass ceramic.

On the contrary, the results of this study disagreed with Gluce et al ${ }^{(24)}$ and Gunal et al. (25) who concluded that Vita Suprinity had lower translucency than that of lithium disilicate which in turn affects the degree of conversion and water sorption. They assumed that this may be due to lithium disilicates crystal structure $(1 \mu \mathrm{m})$ that is higher than that of zirconia reinforced lithium silicate $(0.5 \mu \mathrm{m})$. In addition, lithium disilicate consist 1 type of crystals and zirconia reinforced lithium silicate consists 2 types of crystal structure. It was reported that the difference in translucency is caused by the differences among the crystalline structures and can be interpreted as a difference in the form and volume of the crystals inside the glass ceramic. This difference in results may be attributed to the coffee thermocycling that applied in their study.

As regards for the different thicknesses tested, there was no significant difference between them where the degree of conversion of the resin cement 
was adequate in both thicknesses. The results of this study came in agreement with several previous studies where Ganjkar et al. ${ }^{(26)}$ and Seok-Hwan et al. ${ }^{(27)}$ stated that the ceramic thickness doesn't have a significant effect on the degree of conversion up to $2 \mathrm{~mm}$ thickness consequently not affecting the water sorption values.

In addition, Runnacles et al. ${ }^{(16)}$ reported similar results stating that up to $1 \mathrm{~mm}$ thickness had no effect on the degree of conversion in the light cured resin cement. On the other hand, Maraghy et al ${ }^{(21)}$ reported in their study that there was a significant difference in degree of conversion between $0.5 \mathrm{~mm}$ and $1 \mathrm{~mm}$ ceramic thickness. The difference between the reported results may be owed to the difference in the translucencies used in both studies where IPS emax CAD low translucency was used in their study and IPS emax CAD high translucency was used in the present study.

\section{CONCLUSION}

There is a relationship between ceramic type including zirconia reinforced lithium silicates and lithium disilicate ceramic materials and water sorption of the resin cement while different ceramic thicknesses up to $1 \mathrm{~mm}$ did not affect the water sorption of the light cured resin cement.

\section{REFERENCES}

1. Wendler M, Belli R, Petschelt A, et al. Chairside CAD/CAM materials. Part 2: Flexural strength testing. Dent Mater. 2017;33(1):99-109.

2. Schwindling FS, Rues S, Schmitter M. Fracture resistance of glazed, full-contour ZLS incisor crowns. J Prosthodont Res. 2017;61(3):344-349.

3. Elsaka SE, Elnaghy AM. Mechanical properties of zirconia reinforced lithium silicate glass-ceramic. Dent Mater. 2016;32(7):908-914

4. Aguiar TR, De Oliveira M, Arrais CAG, Ambrosano GMB, Rueggeberg F, Giannini M. The effect of photopolymerization on the degree of conversion, polymerization kinetic, biaxial flexure strength, and modulus of self-adhesive resin cements. J Prosthet Dent. 2015;113(2):128-134.
5. Gregor L, Bouillaguet S, Onisor I, Ardu S, Krejci I, Rocca GT. Microhardness of light- and dual-polymerizable luting resins polymerized through 7.5-mm-thick endocrowns. J Prosthet Dent. 2014;112(4):942-948.

6. Heo YJ, Lee GH, Park JK, et al. Effect of energy density on low-shrinkage composite resins: Diode-pumped solid state laser versus quartz-tungsten-halogen light-curing unit. Photomed Laser Surg. 2013;31(1):28-35.

7. Schulze KA, Marshall SJ, Gansky SA, Marshall GW. Color stability and hardness in dental composites after accelerated aging. Dent Mater. 2003;19(7):612-619.

8. Tanoue N, Koishi Y, Atsuta M, Matsumura H. Properties of dual-curable luting composites polymerized with single and dual curing modes. J Oral Rehabil. 2003;30(10):10151021.

9. Petropoulou A, Vrochari AD, Hellwig E, Stampf S, Polydorou O. Water sorption and water solubility of selfetching and self-adhesive resin cements. J Prosthet Dent. 2015;114(5):674-679.

10. MESE A, BURROW MF, TYAS MJ. Sorption and solubility of luting cements in different solutions. Dent Mater J. 2008;27(5):702-709.

11. Hersek NE, Canay Ş. In vivo solubility of three types of luting cement. Quintessence Int (Berl). 1996;27(3):211-216.

12. Yiu CKY, King NM, Carrilho MRO, et al. Effect of resin hydrophilicity and temperature on water sorption of dental adhesive resins. Biomaterials. 2006;27(9):1695-1703.

13. Mathias C, Vitória LA, Gomes RS, Cavalcanti AN, Mathias P. Influence of Ceramic Laminate Veneer Thickness on Sorption and Solubility of Light-Cured Resin Cement. Int J Dent Oral Sci. 2017;(February):422-426.

14. Franken P, Rodrigues SB, Collares FM, Samuel SMW, Leitune VCB. Influence of N-(2-hydroxyethyl)acrylamide addition in light- and dual-cured resin cements. J Dent. 2019;90(October):103-208.

15. Zaghloul KIK, Mohsen CA. Translucency of cad/cam veneers using different internal relief spaces and luting cement shades. Indian J Public Heal Res Dev. 2020;11(6):1323-1329.

16. Runnacles P, Correr GM, Filho FB, Gonzaga CC, Furuse AY. Degree of conversion of a resin cement light-cured through ceramic veneers of different thicknesses and types. Braz Dent J. 2014;25(1):38-42.

17. Lavergne F, Belhadi R, Carriat J, Ben Fraj A. Effect of nano-silica particles on the hydration, the rheology and the strength development of a blended cement paste. Cem Concr Compos. 2019;95:42-55. 
18. Sokolowski G, Szczesio A, Bociong K, et al. Dental resin cements-The influence of water sorption on contraction stress changes and hydroscopic expansion. Materials (Basel). 2018;11(6).

19. Leal CL, Queiroz AP V, Foxton RM, Mathias SAP, Cavalcanti AN. Water Sorption and Solubility of Luting Agents Used Under Ceramic Laminates With Different Degrees of Translucency. 2016:141-148.

20. Awad D, Stawarczyk B, Liebermann A, Ilie N. Translucency of esthetic dental restorative CAD/CAM materials and composite resins with respect to thickness and surface roughness. J Prosthet Dent. 2015;113(6):534-540.

21. Maraghy MAR, Zohdy MM, Wahsh MM. Degree of Conversion of Light Cured Resin Cements Polymerized under Two Thicknesses of Different Lithium Silicate Ceramics. J Dent Oral Sci. 2020;2(1):1-12.

22. Caprak YO, Turkoglu P. Does the Translucency of Novel Monolithic CAD / CAM Materials Affect Resin Cement Polymerization with Different Curing Modes ? 2018:1-8.
23. Bahgat SFA, Basheer RR and El Sayed SM, Effect of zirconia addition to lithium disilicate ceramic on translucency and bond strength using different adhesive strategies EDJ. 2015;61:4519-4533.

24. Gülce M, Alp G, Johnston WM, Yilmaz B. Effect of thickness on optical properties of monolithic CAD-CAM ceramics. jdent 2018.

25. Gunal B, Ulusoy MM. Optical properties of contemporary monolithic CAD-CAM restorative materials at different thicknesses. J Esthet Restor Dent. 2018;30(5):434-441.

26. Ganjkar MH, Heshmat H, Ahangari RH. Evaluation of the Effect of Porcelain Laminate Thickness on Degree of Conversion of Light Cure and Dual Cure Resin Cements Using FTIR. 2017;18(1):30-36.

27. Cho SH, Lopez A, Berzins DW, Prasad S and Ahn KW. Effect of Different Thicknesses of Pressable Ceramic Veneers on Polymerization of Light-cured and Dualcured Resin Cements J Contemp Dent Pract. 2015;16(5): 347-352. 